\title{
Risk variants of the a-synuclein locus and REM sleep behavior disorder in Parkinson's disease: a genetic association study
}

\author{
Kari Anne Bjørnarå ${ }^{1 *+} \mathbb{D}$, Lasse Pihlstrøm ${ }^{2,3+}$, Espen Dietrichs $s^{2,3}$ and Mathias Toft ${ }^{2,3}$
}

\begin{abstract}
Background: Parkinson's disease is a heterogeneous disorder where genetic factors may underlie clinical variability. Rapid eye movement sleep behavior disorder (RBD) is a parasomnia strongly linked to synucleinopathies, including Parkinson's disease. We hypothesized that SNCA variants conferring risk of Parkinson's disease would also predispose to an RBD phenotype.

Methods: We assessed possible RBD (pRBD) status using the RBD screening questionnaire and investigated known susceptibility variants for Parkinson's disease located in the a-synuclein (SNCA) and tau (MAPT) gene loci in 325 Parkinson's disease patients. Associations between genetic risk variants and RBD were investigated by logistic regression, and an independent dataset of 382 patients from the Parkinson's Progression Marker Initiative (PPMI) study was used for replication.

Results: pRBD was associated with rs3756063 located in the $5^{\prime}$ region of SNCA (two-sided $p=0.018$, odds ratio 1.44). We replicated this finding in the PPMI dataset (one-sided $p=0.036$, odds ratio 1.35) and meta-analyzed the results (two-sided $p=0.0032$, odds ratio 1.40). The Parkinson's disease risk variant in the $3^{\prime}$ region of SNCA and the MAPT variant showed no association with $\mathrm{PRBD}$.

Conclusions: Our findings provide proof of principle that a largely stable, dichotomous clinical feature of Parkinson's disease can be linked to a specific genetic susceptibility profile. Indirectly, it also supports the hypothesis of RBD as relevant marker for a distinct subtype of the disorder.
\end{abstract}

Keywords: SNCA, a-synuclein; genetics, REM sleep behavior disorder, RBD, Parkinson's disease

\section{Background}

Parkinson's disease (PD) is a highly heterogeneous disorder. The prospect of linking clinical subtypes of PD to specific disease mechanisms and pathways would represent an important advance for optimization of clinical trials and, eventually, individualized treatment. Differences in the genetic basis of disease are often assumed to underlie variability in symptoms. Yet where large case-control studies have identified a range of common loci affecting disease risk for sporadic PD, the evidence for genetic modulation of the clinical presentation is currently scarce.

\footnotetext{
* Correspondence: Kari.Anne.Bjornara@vestreviken.no

${ }^{\dagger}$ Equal contributors

'Department of Neurology, Drammen Hospital, Vestre Viken Hospital Trust, Dronninggata 21, 3004 Drammen, Norway

Full list of author information is available at the end of the article
}

RBD is a parasomnia characterized by absence of muscle atonia during REM sleep. Affected individuals typically present vigorous motor activity and vocalization while they "live out their dreams". Patients with idiopathic RBD are at high risk of developing neurodegenerative disease, in particular those disorders that are histopathologically characterized by $\alpha$-synuclein aggregates [1]. The parasomnia is a frequent clinical feature in all synucleinopathies, including PD, where RBD affects about half of the patients and has been associated with more severe dysautonomia, cognitive impairment and increased risk of dementia $[2,3]$. In contrast, RBD is rare in tauopathies like Alzheimer's disease (AD) and progressive supranuclear palsy [4].

Synuclein and tau pathologies are neuropathological findings that despite considerable overlap reflect distinct disease processes. Both the $\alpha$-synuclein $(S N C A)$ and the 
tau $(M A P T)$ gene regions are major susceptibility loci for sporadic PD, corroborated in several large GWAS [5]. Since RBD is strongly associated to synucleinopathies, we hypothesized that SNCA variants conferring risk of PD would also predispose to an RBD phenotype. Based on insights from neuropathology, we did not expect a similar effect of MAPT variability. We assessed RBD status and investigated SNPs representing SNCA and MAPT association signals in 325 PD patients from Norway, using data from PPMI as a replication set.

\section{Methods}

\section{Subjects}

A total of 325 patients diagnosed with PD by the UK Brain Bank criteria [6] were recruited from Oslo University Hospital and Drammen Hospital in South-East Norway. Patients of non-Scandinavian origin or with a known monogenic form of PD were excluded. The study was approved by the Regional Committee for Medical and Health Research Ethics in South-East Norway and all participants gave informed, written consent.

\section{Assessment of REM sleep behavior disorder}

In our study, we used the RBD screening questionnaire (RBDSQ) to diagnose possible RBD (pRBD, i.e. history of dream enactment without polysomnographically confirmed REM sleep without atonia). RBDSQ is also included as part of the PPMI test battery. The RBDSQ consists of 13 yes/no questions, where six or more positive answers correspond to the optimal cut-off for detecting pRBD in PD. The questionnaire has been validated in the PD population with a sensitivity of $84 \%$ and a specificity of $96 \%$ [7]. Demographic characteristics and medication use of the included PD patient groups with and without pRBD are summarized in Table 1.

\section{Genotyping}

We selected markers representing significant GWAS signals from our two loci of interest, including one SNP from MAPT (rs2942168) and three from SNCA (rs356165, rs3756063 and rs2245801). Across previous large-scale studies, the most significant $S N C A$ variants have consistently been located near the 3' end of the gene, tagged by rs356165 [8, 9]. However, in stepwise conditional analysis, an independent, secondary association signal has been identified in the 5 ' region [10]. In studies with largely overlapping samples, different 5' SNPs in only moderate linkage disequilibrium (LD) have been reported as secondary top-hits (rs2245801and rs7681154; $\mathrm{r}^{2}=0.22$, D'=0.99), and it is currently unclear whether these represent distinct or similar underlying functional variation $[5,8]$. For reasons related to assay availability, rs7681154 was substituted by rs3756063 $\left(\mathrm{r}^{2}=0.97\right)$ and $\mathrm{rs} 1372520$ was used as a proxy for $\mathrm{rs} 2245801\left(\mathrm{r}^{2}=0.88\right)$ in a subset of samples.

DNA was extracted from whole blood. Genotyping was performed using KASPar or TaqMan assays on a ViiA7 instrument (Life Technologies, Foster City, CA, USA). The genotyping rate was $>95 \%$ for all SNPs, and we observed no departure from Hardy-Weinberg equilibrium $(p<0.01)$.

\section{Replication in the PPMI dataset}

Data on demographics, RBDSQ and genetic data genotyped on the NeuroX array were downloaded from the PPMI website. We identified 382 genotyped PD patients who had completed RBDSQ on at least one occasion and applied the same cutoff as in the discovery dataset to each patient's median score. Genotype data was filtered by removing variants with minor allele frequency $<0.01$, genotype call rate $<0.95$ or Hardy-Weinberg equilibrium $p>1 * 10^{-6}$, and imputed using the Michigan Imputation Server with data from the Haplotype Reference Consortuim as reference and otherwise default parameters [11], The SNP nominated as significant from the Norwegian dataset (rs3756063) was successfully imputed with a high quality score $\left(r^{2}=0.999\right)$. We further calculated principal components for the PPMI dataset based on a subset of

Table 1 Demographic characteristics and medication use

\begin{tabular}{|c|c|c|c|c|}
\hline & Total (325) & pRBD (141) & Non-RBD (184) & $p$ value \\
\hline Male:female & $213: 112(65.5)$ & $106: 35(75.2)$ & $107: 77(58.2)$ & $<0.01$ \\
\hline Age, years & $64.9(9.4)$ & $64.1(9.4)$ & $65.4(9.4)$ & 0.45 \\
\hline Disease duration, years & $9.2(6.9)$ & $10.9(7.2)$ & $7.7(6.4)$ & 0.02 \\
\hline Levodopa yes:no & $270: 53(83.6)$ & $124: 15(87.9)$ & $146: 38(79.3)$ & 0.50 \\
\hline Agonist yes:no & 213:110 (65.9) & $80: 59(56.7)$ & $133: 51(72.3)$ & 0.03 \\
\hline SSRI/SNRI yes:no & $52: 269(16.2)$ & $32: 106(22.7)$ & 20:163 (10.9) & 0.02 \\
\hline Benzodiazepine yes:no & 42:278 (15.1) & 22:115 (15.6) & 20:163 (10.9) & 0.20 \\
\hline Clonazepam yes:no & 19:302 (5.8) & $12: 126(8.5)$ & $7: 176(3.8)$ & 0.50 \\
\hline
\end{tabular}

Demographic data and medication use in PD patients with and without pRBD, respectively. Numbers in parentheses refers to percentage, except for age and disease duration, where it refers to standard deviation. $P$ values refer to binary logistic regression analyses

Abbreviations: pRBD possible REM-sleep behavior disorder, SSRI selective serotonin reuptake inhibitor, SNRI serotonin and norepinephrine reuptake inhibitor 
common, linkage disequilibrium (LD)-pruned SNPs, using minor allele frequency $>0.05$ and pairwise $r^{2}<0.5$ as thresholds.

\section{Statistical analysis and power considerations}

Statistical analyses were performed in PLINK [12] and SPSS v.21 (Armonk, New York, USA). Associations between SNP alleles and RBD status were assessed by logistic regression, including age and gender as covariates. For the replication analysis in PPMI data we also included the top four principal components in the regression model.

Notably, association studies of common symptoms within a disease group will typically have higher power compared to traditional case-control studies, due to the high prevalence of both outcomes. Based on 141 pRBD vs 184 non-RBD samples, we estimated the statistical power to detect an effect size of odds ratio 1.3 for a variant of allele frequency 0.5 to be $90 \%$ at $p<0.05$, using the Genetic Power Calculator (zzz.bwh.harward.edu/gpc/cc2.html). Given the context of an exploratory study with a limited number of independent statistical tests, we set a threshold of $p<0.05$ to nominate variants from the discovery analysis for further replication. For the one SNP assessed for replication in PPMI data, we hypothesized a consistent direction of effect, allowing for a one-sided $p$-value, and in the final meta-analysis, we set the significance threshold to $p<0.0125$, adjusting for a total of 4 SNPs investigated in the study.

\section{Results}

pRBD was more frequent in men $(p<0.01)$. The association results for SNPs versus pRBD status in PD patients, adjusted for age and gender by binary logistic regression, are shown in Table 2. pRBD was significantly associated with rs3756063 located in the $5^{\prime}$ region of SNCA ( $p=0.018$, odds ratio 1.44). We observed no association for the other $S N C A$ variants or MAPT. Repeating the same analysis in the independent PPMI dataset for rs3756063, we replicated the same result with marginal significance (one-sided $p=$ 0.036 , odds ratio 1.35), yet strikingly similar effect size and allele frequencies across groups. Meta-analysis of the results under a fixed effects model showed a two-sided $p$-value of 0.0032 and odds ratio 1.40 . There was no indication of heterogeneity of effects across studies (heterogeneity index $\mathrm{I}^{2}=0.00$ ).

\section{Discussion}

RBD is highly prevalent in the PD population, and the present study is to our knowledge the first to explore genetic risk factors for pRBD within this context. The idiopathic form of the parasomnia is rare, yet intensively researched as one of the strongest known clinical markers of prodromal neurodegenerative disease. A recent case-control study investigated top-hits from PD GWAS in 261 patients with idiopathic RBD and 379 healthy controls, reporting significant associations with $M A P T$ and $S C A R B 2$ [13]. Assuming that MAPT could conceivably be an equally strong risk factor for PD without RBD, their findings are not necessarily in conflict with the lack of association with MAPT seen in the present study. Although other loci were negative, the authors noted non-significant odds ratios that resembled those seen in PD for several other signals, including $S N C A$. Importantly, the secondary SNCA GWAS signal highlighted in the present study was not investigated.

Knowledge about the contributions of common genetic variants to clinical variability in sporadic PD is currently limited. SNCA variability has been reported to be associated with both motor progression and cognitive outcomes, but results have varied across studies [14-16]. Evidence is currently accumulating both for other single loci and for the cumulative genetic risk burden with respect to their influence on motor and cognitive progression, although most published studies are moderate in sample size [15, 17-19]. Where motor symptoms and cognition are

Table 2 Association results for pRBD with investigated SNCA and MAPT variants

\begin{tabular}{|c|c|c|c|c|c|c|c|}
\hline dbSNP ID & Locus & Alleles & Frequency pRBD & Frequency Non-RBD & SE & OR & $p$ value \\
\hline \multicolumn{8}{|c|}{ Norwegian discovery dataset $N=141$ pRBD-PD / 184 non-RBD-PD } \\
\hline rs356165 & SNCA & $\mathrm{G} / \mathrm{A}$ & 0.42 & 0.42 & 0.163 & 1.07 & 0.76 \\
\hline rs3756063 & SNCA & $C / G$ & 0.59 & 0.48 & 0.155 & 1.44 & 0.018 \\
\hline rs2245801 & SNCA & $\mathrm{T} / \mathrm{C}$ & 0.11 & 0.14 & 0.261 & 0.79 & 0.36 \\
\hline rs2942168 & MAPT & $\mathrm{T} / \mathrm{C}$ & 0.14 & 0.11 & 0.239 & 1.27 & 0.33 \\
\hline \multicolumn{8}{|c|}{ PPMI replication dataset: $N=106$ pRBD-PD / 276 non-RBD-PD } \\
\hline rs3756063 & SNCA & $C / G$ & 0.56 & 0.49 & 0.168 & 1.35 & $0.036^{\mathrm{a}}$ \\
\hline \multicolumn{5}{|c|}{ Meta-analysis across both datasets for rs3756063: } & 0.114 & 1.40 & 0.0032 \\
\hline
\end{tabular}

The association between SNPs and RBD was tested in a logistic regression model, including age and gender as covariates. The first allele listed is the effect allele Abbreviations: SNP single-nucleotide polymorphism, PD Parkinson's disease, $p R B D$ possible REM-sleep behavior disorder, OR odds ratio, SE standard error ane-sided p-value 
strongly associated with age and disease duration, RBD is arguably a more distinctly dichotomous clinical feature, showing weaker association to most demographic data. RBD occurs only in a proportion of PD patients and may appear even before motor symptoms [4]. RBD has been interpreted as a probable indicator of more extensive neuropathology in PD [2,3]. Our finding suggests a specific genetic architecture predisposing to PD with $\mathrm{pRBD}$, strengthening the rationale for $\mathrm{RBD}$ as marker for a dichotomy into PD subtypes.

The associated variant is located in the $5^{\prime}$ region of SNCA and represents a secondary GWAS signal, emerging as significant when conditioning on the $3^{\prime}$ top-hit SNP. While the causal mechanisms linking genetic variation to disease processes on a molecular scale are currently unknown, they are likely to involve complex regulatory networks specific to tissue subpopulations [20,21]. Possibly, the discrepancy between the $3^{\prime}$ and $5^{\prime}$ variants' effect on pRBD observed in the present study might reflect regional differences in neuronal SNCA regulation, indicating that multiple genetic risk variants at the same locus may carry a distinct pathogenic relevance.

We find that disease duration is longer in PD patients with pRBD. This is seen in some studies [22, 23]. However, several studies have shown that RBD can precede motor symptoms by decades, and thus the impact of disease duration on RBD symptoms is still unclear. Additionally, use of antidepressants is higher in the pRBD group. Antidepressants may increase the frequency of RBD, and could thus be a confounder [24].

A limitation of our study is the lack of polysomnography, as we are not able to identify RBD with absolute certainty. However, RBDSQ is widely used in larger studies, including the PPMI. Furthermore, according to the DSM-5 criteria for RBD, a suggestive history is enough to diagnose RBD in patients with an established synucleinopathy. Mean disease duration is 9 years, which supports the clinical diagnose of PD.

\section{Conclusion}

pRBD was significantly associated with a known GWAShit variant in the $5^{\prime}$ region of $S N C A$, highlighting how distinctive clinical features in PD may depend on the genetic basis of disease.

Our study provides proof of principle for the integration of clinical, neuropathological and genetic observations to gain novel insights into the heterogeneous nature of sporadic PD. We anticipate a range of similar genotypephenotype correlations emerging in future studies, mapping the clinicogenetic landscape of PD, with important implications for future therapeutic strategies.

\section{Abbreviations}

GWAS: Genome-wide association study; PD: Parkinson's disease; PPMI: Parkinson's Progression Marker Initiative; pRBD: Possible REM sleep behavior disorder;
RBD: REM sleep behavior disorder; RBDSQ: RBD Screening Questionnaire; REM: Rapid eye movement; SNP: Single-nucleotide polymorphism

\section{Acknowledgements}

Data used in the preparation of this article were obtained from the Parkinson's Progression Markers Initiative (PPMI) database (www.ppmi-info.org/data). For up-to -date information of the study, visit www.ppmi-info.org.

\section{Availability of data and materials}

The Norwegian dataset generated and analyzed during the current study are not publicly available due to privacy constraints relating to the ethical approval and informed consent signed by the participants, but is available from the corresponding author on reasonable request. Data from the PPMI study used for replication are available to researchers upon request, see www.ppmi-info.org.

\section{Authors' contributions}

KAB and MT collected samples and clinical data. MT, LP and KAB designed the study. LP performed genotyping. LP and KAB performed statistical analyses. MT and ED provided supervision. KAB and LP drafted the manuscript. All authors contributed to critical revision and approved the final manuscript.

\section{Ethics approval and consent to participate}

The study was approved by the Regional Committee for Medical and Health Research Ethics in South-East Norway and all participants gave informed, written consent

\section{Consent for publication}

Not applicable

\section{Competing interests}

This work was supported by grants from Vestre Viken Hospital Trust, the Norwegian Parkinson's Disease Association, Reberg's Legacy, the Norwegian Neurological Society, Anna Garaas, South-Eastern Norway Regional Health Authority and the Research Council of Norway. PPMI is sponsored and partially funded by the Michael J. Fox Foundation for Parkinson's Research (MJFF). Other funding partners include a consortium of industry players, non-profit organizations and private individuals, including Abbott, Avid, Biogen Idec, Covance, Elan, GlaxoSmithKline, Lilly, Merck, UCB, F. Hoffman-La Roche Ltd., GE Healthcare, Genentech, and Pfizer Inc. Industry partners are contributing to PPMI through financial and in-kind donations and are playing a lead role in providing feedback on study parameters through the Industry Scientific Advisory Board (ISAB). For up-to-date information on the study, visit www.ppmi-info.org. The funders had no role in study design, collection, analysis and interpretation of data, writing of the article or the decision to submit the article for publication. The authors declare that they have no competing interests.

\section{Publisher's Note}

Springer Nature remains neutral with regard to jurisdictional claims in published maps and institutional affiliations.

\section{Author details}

'Department of Neurology, Drammen Hospital, Vestre Viken Hospital Trust, Dronninggata 21, 3004 Drammen, Norway. ${ }^{2}$ Department of Neurology, Oslo University Hospital, Oslo, Norway. ${ }^{3}$ Institute of Clinical Medicine, University of Oslo, Oslo, Norway.

Received: 12 October 2017 Accepted: 8 February 2018

Published online: 21 February 2018

\section{References}

1. Schenck CH, Boeve BF, Mahowald MW. Delayed emergence of a parkinsonian disorder or dementia in $81 \%$ of older men initially diagnosed with idiopathic rapid eye movement sleep behavior disorder: a 16-year update on a previously reported series. Sleep Med. 2013;14(8):744-8.

2. Postuma RB, Bertrand JA, Montplaisir J, Desjardins C, Vendette M, Rios Romenets S, Panisset M, Gagnon JF. Rapid eye movement sleep behavior disorder and risk of dementia in Parkinson's disease: a prospective study. Mov Disord. 2012;27(6):720-6. 
3. Romenets SR, Gagnon JF, Latreille V, Panniset M, Chouinard S, Montplaisir J, Postuma RB. Rapid eye movement sleep behavior disorder and subtypes of Parkinson's disease. Mov Disord. 2012;27(8):996-1003.

4. Gagnon JF, Postuma RB, Mazza S, Doyon J, Montplaisir J. Rapid-eyemovement sleep behaviour disorder and neurodegenerative diseases. Lancet Neurol. 2006:5(5):424-32.

5. Nalls MA, Pankratz N, Lill CM, Do CB, Hernandez DG, Saad M, DeStefano AL, Kara E, Bras J, Sharma M, et al. Large-scale meta-analysis of genome-wide association data identifies six new risk loci for Parkinson's disease. Nat Genet. 2014;46(9):989-93.

6. Gibb WR, Lees AJ. The relevance of the Lewy body to the pathogenesis of idiopathic Parkinson's disease. J Neurol Neurosurg Psychiatry. 1988;51(6): 745-52.

7. Nomura T, Inoue $Y$, Kagimura T, Uemura $Y$, Nakashima K. Utility of the REM sleep behavior disorder screening questionnaire (RBDSQ) in Parkinson's disease patients. Sleep Med. 2011;12(7):711-3.

8. International Parkinson's Disease Genomics Consortium, Wellcome Trust Case Control Consortium. A two-stage meta-analysis identifies several new loci for Parkinson's disease. PLoS Genet. 2011;7(6):e1002142.

9. Lill CM, Roehr JT, McQueen MB, Kavvoura FK, Bagade S, Schjeide BM, Schjeide LM, Meissner E, Zauft U, Allen NC, et al. Comprehensive research synopsis and systematic meta-analyses in Parkinson's disease genetics: the PDGene database. PLoS Genet. 2012;8(3):e1002548.

10. Spencer CC, Plagnol V, Strange A, Gardner M, Paisan-Ruiz C, Band G, Barker RA, Bellenguez C, Bhatia K, Blackburn H, et al. Dissection of the genetics of Parkinson's disease identifies an additional association $5^{\prime}$ of SNCA and multiple associated haplotypes at 17q21. Hum Mol Genet. 2011;20(2):345-53.

11. Loh PR, Danecek P, Palamara PF, Fuchsberger C, A Reshef $Y, K$ Finucane $H$ Schoenherr S, Forer L, McCarthy S, Abecasis GR, et al. Reference-based phasing using the haplotype reference consortium panel. Nat Genet. 2016; 48(11):1443-8.

12. Purcell $S$, Neale B, Todd-Brown K, Thomas L, Ferreira MA, Bender D, Maller J, Sklar P, de Bakker PI, Daly MJ, et al. PLINK: a tool set for whole-genome association and population-based linkage analyses. Am J Hum Genet. 2007; 81(3):559-75.

13. Gan-Or Z, Girard SL, Noreau A, Leblond CS, Gagnon JF, Arnulf I, Mirarchi C, Dauvilliers $Y$, Desautels A, Mitterling T, et al. Parkinson's disease genetic loci in rapid eye movement sleep behavior disorder. J Mol Neurosci. 2015; 56(3):617-22.

14. Ritz B, Rhodes SL, Bordelon Y, Bronstein J. Alpha-Synuclein genetic variants predict faster motor symptom progression in idiopathic Parkinson disease. PLoS One. 2012;7(5):e36199.

15. Mata IF, Leverenz JB, Weintraub D, Trojanowski JQ, Hurtig HI, Van Deerlin VM, Ritz B, Rausch R, Rhodes SL, Factor SA, et al. APOE, MAPT, and SNCA genes and cognitive performance in Parkinson disease. JAMA Neurol. 2014; 71(11):1405-12.

16. Cooper CA, Jain N, Gallagher MD, Weintraub D, Xie SX, Berlyand Y, Espay AJ, Quinn J, Edwards KL, Montine T, et al. Common variant rs356182 near SNCA defines a Parkinson's disease endophenotype. Ann Clin Transl Neurol. 2017; 4(1):15-25.

17. Nombela C, Rowe JB, Winder-Rhodes SE, Hampshire A, Owen AM, Breen DP, Duncan GW, Khoo TK, Yarnall AJ, Firbank MJ, et al. Genetic impact on cognition and brain function in newly diagnosed Parkinson's disease: ICICLE-PD study. Brain. 2014;137(Pt 10):2743-58.

18. Davis AA, Andruska KM, Benitez BA, Racette BA, Perlmutter JS, Cruchaga C. Variants in GBA, SNCA, and MAPT influence Parkinson disease risk, age at onset, and progression. Neurobiol Aging. 2016;37:209. e201-207

19. Pihlstrom L, Morset KR, Grimstad E, Vitelli V, Toft M. A cumulative genetic risk score predicts progression in Parkinson's disease. Mov Disord. 2016; 31(4):487-90.

20. Ko Y, Ament SA, Eddy JA, Caballero J, Earls JC, Hood L, Price ND. Cell type-specific genes show striking and distinct patterns of spatial expression in the mouse brain. Proc Natl Acad Sci U S A. 2013;110(8):3095-100.

21. Ramasamy A, Trabzuni D, Guelfi S, Varghese V, Smith C, Walker R, De T, Coin $L$, de Silva $R$, Cookson MR, et al. Genetic variability in the regulation of gene expression in ten regions of the human brain. Nat Neurosci. 2014;17(10): 1418-28.

22. Bjornara KA, Dietrichs E, Toft M. Longitudinal assessment of probable rapid eye movement sleep behaviour disorder in Parkinson's disease. Eur J Neurol. 2015;22(8):1242-4
23. Sixel-Doring F, Trautmann E, Mollenhauer B, Trenkwalder C. Associated factors for REM sleep behavior disorder in Parkinson disease. Neurology. 2011;77(11):1048-54.

24. Boeve BF, Silber MH, Saper CB, Ferman TJ, Dickson DW, Parisi JE, Benarroch EE, Ahlskog JE, Smith GE, Caselli RC, et al. Pathophysiology of REM sleep behaviour disorder and relevance to neurodegenerative disease. Brain. 2007; 130(Pt 11):2770-88.

\section{Submit your next manuscript to BioMed Central and we will help you at every step:}

- We accept pre-submission inquiries

- Our selector tool helps you to find the most relevant journal

- We provide round the clock customer support

- Convenient online submission

- Thorough peer review

- Inclusion in PubMed and all major indexing services

- Maximum visibility for your research

Submit your manuscript at www.biomedcentral.com/submit 